\title{
Stability of three-dimensional self-trapped beams with a dark spot surrounded by bright rings of varying intensity
}

\author{
Javid Atai and Yijiang Chen \\ Optical Sciences Centre, The Australian National University, Canberra, Australian Capital Territory 0200, Australia \\ José Maria Soto-Crespo \\ Instituto de Optica, Consejo Superior de Investigaciones Científicas, Serrano 121, 28006 Madrid, Spain
}

(Received 7 September 1993)

\begin{abstract}
We analytically and numerically examine the stability of three-dimensional self-trapped beams with a dark spot surrounded by bright rings of varying intensity in a uniform saturable self-focusing medium. It is shown that the fundamental bound state of the family is stable to a symmetric perturbation but unstable to an asymmetric perturbation (that breaks the azimuthal symmetry of the beam, i.e., transverse instabilities). The higher-order states are also found to display transverse (modulation) instabilities. The development of the instabilities is shown to lead to the emission of filaments which spiral away from the center of the dark spot as stable entities.
\end{abstract}

PACS number(s): 42.65.-k, 42.25.Bs, 42.50.Rh

Self-trapping of optical beams in a nonlinear medium has been a subject of interest and investigated extensively over the last three decades both experimentally and theoretically $[1-15]$. In their pioneering works, Chiao, Garmire, and Townes [1] and Haus [2] demonstrated that in a Kerr-law nonlinear medium, a cylindrically symmetric beam with the maximum intensity at the center can be a three-dimensional self-trapped beam pattern (which in theory remains unchanged with the propagation distance). However, in practice it may not be the case, as this family of bound-state solutions is not stable against a symmetric perturbation [3]. Above the critical trapping power the beam focuses, and below the critical trapping power the beam diffracts. In a saturable nonlinear medium (as is often the case in practice), the trapped beams demonstrate quite different stability characteristics. The fundamental state of the family becomes stable (against both symmetric and asymmetric perturbations) [9], whereas the higher-order states are unstable against the perturbation, which breaks the azimuthal symmetry of the beams (transverse instabilities) [10]; and these stability results, which were predicted from the linear stability analysis, have been confirmed by direct numerical simulations of the nonlinear wave equation [15].

The self-trapped beam patterns discovered in Refs. $[1,2]$ are not the only family of trapped light patterns [with a linear polarization in the $x$ or $y$ direction, which are approximations to the hybrid trapped light patterns $\left(\mathrm{HE}_{1 m}\right)$ resulting from Maxwell's equations]. There actually exists another family of self-trapped light pattern characterized by a dark spot surrounded by bright rings of varying intensity (called TE patterns) first reported in Ref. [16] for Kerr-law nonlinearity and late in Ref. [17] for a saturable nonlinearity. Similar to the trapped light patterns with maximum intensity at the center, in the Kerr-law medium this family of TE self-trapped light patterns is unstable against a symmetric perturbation [18]; the beam focuses or diffracts when the power is greater or smaller than the trapping power. In a saturable nonlinear medium, the fundamental state of this TE family was shown to be stable against symmetric perturbation, based on the optical force argument [17]. However, the question of the stability of this fundamental state against the perturbation that breaks the symmetry of the beam (transverse stability) and the stability of higher-order states has been left unaddressed.

Here, we examine the stability (including the transverse or modulation stability) of the TE family of the self-trapped beams both by linear stability analysis and by direct numerical simulations on the wave equation. Our linear stability analysis shows that the fundamental bound state of this TE family of self-trapped beams is stable against a symmetric perturbation, confirming the work of Ref. [17]; however, it also reveals that this fundamental bound state is unstable against asymmetric perturbation that breaks the azimuthal symmetry of the beam. The higher-order states of the family are found to display transverse instabilities as well. And all the results obtained from the linear stability analysis are corroborated by direct numerical simulations which unveil nonlinear dynamics of various evolution patterns of spiralling filaments emitted from perturbed bound states.

The propagation of a light beam in a uniform nonlinear medium follows the vector wave equation [18] which normally involves three field components. One special case is when there is only one azimuthal field component concerned. The vector wave equation then reduces to the scalar wave equation

$i 2 \beta \frac{\partial e_{\phi}}{\partial z}+\nabla_{t}^{2} e_{\phi}-\frac{e_{\phi}}{r^{2}}-\left(\beta^{2}-k^{2} n_{0}^{2}\right) e_{\phi}+k^{2} \Delta n^{2}\left(\left|e_{\phi}\right|^{2}\right) e_{\phi}$

upon invoking the slowly varying approximation with $e_{\phi}=E_{\phi} \exp (-i \beta z)$, where $k$ is the wave number in free space, $n_{0}$ is the linear refractive index, and the nonlinear refractive index, without loss of generality, takes the two-level model $\Delta n^{2}=n_{0} n_{2}\left|e_{\phi}\right|^{2} /\left[1+\left(n_{0} n_{2}\left|e_{\phi}\right|^{2}\right) /\right.$ $\left.\left(n_{\text {sat }}^{2}-n_{0}^{2}\right)\right]$ with $n_{2}$ the nonlinear coefficient and $n_{\text {sat }}$ the 
maximum refractive index in the presence of nonlinearity. In a dimensionless form, $\psi=e_{\phi} \sqrt{n_{0} n_{2} /\left(n_{\mathrm{sat}}^{2}-n_{0}^{2}\right)}$, $Z=z k^{2}\left(n_{\mathrm{sat}}^{2}-n_{0}^{2}\right) / 2 \beta$, and $R=r k \sqrt{n_{\mathrm{sat}}^{2}-n_{0}^{2}}$, Eq. (1) reads

$$
i \frac{\partial \psi}{\partial Z}+\nabla_{T}^{2} \psi-\frac{\psi}{R^{2}}-\gamma^{2} \psi+\frac{\psi^{2}}{1+\psi^{2}} \psi=0
$$

where $\gamma^{2}=\left(\beta^{2} / k^{2}-n_{0}^{2}\right) /\left(n_{\mathrm{sat}}^{2}-n_{0}^{2}\right)$ and $\nabla_{T}^{2}=$ $(1 / R) \partial(R \partial / \partial R) / \partial R+\left(1 / R^{2}\right) \partial^{2} / \partial \phi^{2}$. By setting $\partial \psi / \partial Z=0$ and $\partial^{2} \psi / \partial \phi^{2}=0$, within $0<\gamma<1$, Eq. (2) is shown to admit a family of cylindrically symmetric bound-state solutions (identified by $n=1,2,3, \ldots$ ), the $n$th of which is characterized by a dark spot [i.e., $\psi(0,0)=0$ ] surrounded by $n$ bright rings [17]. The power [defined as $P=\int\left|e_{\phi}\right|^{2} d S=\left(2 \pi / k^{2} n_{0} n_{2}\right) \int \psi^{2} R d R$ ] carried by each bound state increases with increasing $n$. For a fixed $n, P$ increases with increasing $\gamma^{2}$, as shown in Fig. 1(a) for the fundamental bound state, where $P_{c}=15.38 \pi / k^{2} n_{0} n_{2}$ is the critical trapping power for the Kerr-law nonlinearity.

To investigate the stability of a bound state $\psi_{n}$, we seek a perturbed solution of the form

$$
\psi=\psi_{n}+(u+i v) \exp (\delta Z)
$$

which substituted into Eq. (2) leads to

$$
\delta u=-L_{0 n} v, \quad \delta v=L_{1 n} u
$$

upon linearization, where $L_{0 n}=\nabla_{T}^{2}-1 / R^{2}-\gamma^{2}+\psi_{n}^{2} /(1+$ $\left.\psi_{n}^{2}\right)$ and $L_{1 n}=L_{0 n}+2 \psi_{n}^{2} /\left(1+\psi_{n}^{2}\right)^{\frac{1}{2}}$. Note that if $(\delta, u, v)$ is a solution to Eq. (4a), $-\delta, u, v$ are the solutions too. In terms of $u$ alone, the linearized equation (4a) reads

$$
L_{0 n} L_{1 n} u=-\delta^{2} u \text {. }
$$

It can be shown that all the solutions, with $\delta \neq 0$, to Eq. (4) are orthogonal to $\psi_{n}$ (since $\delta\left\langle u \mid \psi_{n}\right\rangle=-\left\langle L_{0 n} v \mid \psi_{n}\right\rangle=$ $-\left\langle v \mid L_{0 n} \psi_{n}\right\rangle=0$ where $\left.\left\langle f_{1} \mid f_{2}\right\rangle=\int f_{1} f_{2}^{*} d S\right)$. So far as the solutions with $\delta \neq 0$ are concerned, Eq. (4) needs to be solved only in the subspace orthogonal to $\psi_{n}$. In this subspace, the inverse operator $L_{0 n}^{-1}$ exists, and for the fundamental bound state the operator $L_{0 n}\left(L_{0 n}^{-1}\right)$ is negative-definite [because all the eigenvalues of $L_{01}$ or $L_{01}^{-1}$ are negative, whereas $L_{0 n}^{-1}(n>1)$ admit positive eigenvalues and are not negative-definite]. Therefore, for

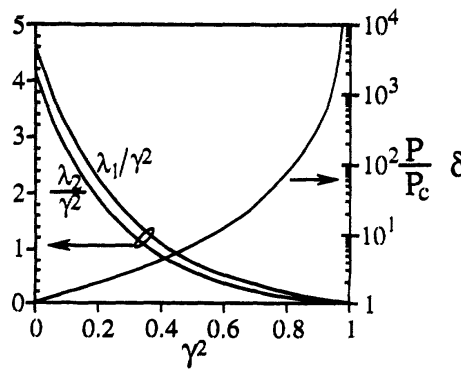

(a)

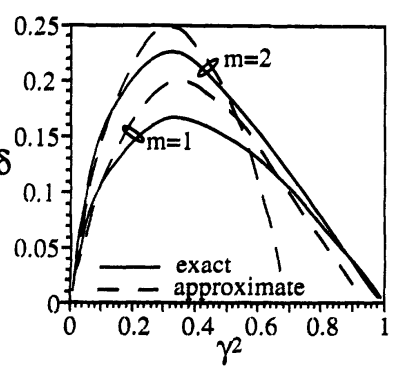

(b)
FIG. 1. (a) Dependence of trapping power $P$ (right scale) of the $n=1$ bound state of the TE family of self-trapped beams on $\gamma^{2}$ and values of $\lambda_{1}$ and $\lambda_{2}$ (left scale). (b) Dependence of the growth rates $\delta$ of the $n=1$ bound state on $\gamma^{2}$. the fundamental bound state the eigenvalues $\delta$ are either real or imaginary and the variational principle can be applied for obtaining the largest value $\delta^{2}$ of Eq. (4b),

$$
\delta^{2}=\max \frac{\left\langle u \mid L_{11} u\right\rangle}{-\left\langle u \mid L_{01}^{-1} u\right\rangle},
$$

where the denominator $-\left\langle u \mid L_{01}^{-1} u\right\rangle$ is a positive quantity ensured by the negative-definiteness of $L_{01}^{-1}$. The value on the numerator of Eq. (5), $G=\left\langle u \mid L_{11} u\right\rangle$, determines whether or not there exists an exponential growth $\delta^{2}>0$ from a perturbation for the fundamental bound state. If $G>0$ real $\delta>0$ exists, implying that the fundamental bound state is unstable, and $G<0$ means that $\delta$ is imaginary and no exponential growing results from a perturbation.

From the method of indeterminate Lagrange multipliers [9] it can be shown that maximization of the quantity $\left\langle u \mid L_{11} u\right\rangle$ in Eq. (5) is equivalent to solve the equation

$$
L_{11} f=\lambda f+q \psi_{1}
$$

for the largest eigenvalue $\lambda$, which together with constant $q$ is determined by the conditions of orthogonality $\left\langle f \mid \psi_{1}\right\rangle=0$ and normalization $\langle f \mid f\rangle=1$. Expanding $f\left(=\sum_{m=1}^{\infty} a_{m} f_{m}\right)$ and $\psi_{1}\left(=\sum_{m=1}^{\infty} c_{m} f_{m}\right)$ of Eq. (6) in the complete set of eigenfunctions $f_{m}$ of the operator $L_{11}$ gives rise to $f=q \sum_{m=1}^{\infty} c_{m} f_{m} /\left(\lambda_{m}-\lambda\right)$. This expansion $f$, substituted into the orthogonality condition $\left\langle f \mid \psi_{1}\right\rangle=0$, leads to an equation for determining $\lambda$,

$$
q g(\lambda)=q \sum_{m=1}^{\infty} \frac{c_{m}^{2}}{\lambda_{m}-\lambda}=0
$$

where $q=0$ when $\lambda=\lambda_{m}$ for asymmetric ( $\phi$ dependent) eigenfunctions. An analysis of symmetric eigenfunctions shows that $L_{11}$ admits only one positive eigenvalue $\lambda_{1}$ [see Fig. 1(a) for $\lambda_{1}$ vs $\gamma$ ] within $0 \leq \gamma<1$. The largest $\lambda=\lambda_{s}$ for the symmetric $f$ (or $\bar{u}$ ) must lie between $\lambda_{1}$ and the largest negative eigenvalue of the symmetric eigenfunction (since $\left\langle f_{1} \mid \psi_{1}\right\rangle \neq 0$ and $f \neq f_{1}$ or $\lambda \neq \lambda_{1}$ ). Equation (7) indicates that $\lambda_{s}>0$ when $g(0)<0$ and $\lambda_{s}<0$ when $g(0)>0$, and this $g(0)$ is related to the power by

$$
\begin{aligned}
g(0)=\sum_{m=1}^{\infty} \frac{c_{m}^{2}}{\lambda_{m}}=\left\langle\psi_{1} \mid L_{11}^{-1} \psi_{1}\right\rangle & =\left\langle\psi_{1} \mid \frac{\partial \psi_{1}}{\partial \gamma^{2}}\right\rangle \\
& =\frac{n_{0} n_{2}}{4 \pi\left(n_{\mathrm{sat}}^{2}-n_{0}^{2}\right)} \frac{d P}{d \gamma^{2}}
\end{aligned}
$$

where the relation $L_{11}\left(\partial \psi_{1} / \partial \gamma^{2}\right)=\psi_{1}$, obtained from differentiating the equation $L_{01} \psi_{1}=0$, has been used in the derivation. From Fig. 1(a) it is seen that within $0 \leq \gamma<1 d P / d \gamma^{2}>0$, leading to $g(0)>0$ or $\lambda_{s}<0$, i.e., no real $\delta$ exists for a symmetric perturbation and in a saturable nonlinear medium the fundamental bound state $\psi_{1}$ is stable against the symmetric perturbation.

Apart from the symmetric eigenfunctions, the operator $L_{11}$ also admits asymmetric eigenvalue solutions (orthogonal to $\left.\psi_{n}\right)$, and the largest eigenvalue $\lambda_{2}=\lambda_{\text {as }}\left(<\lambda_{1}\right)$ of the asymmetric eigenfunctions is found to be pos- 

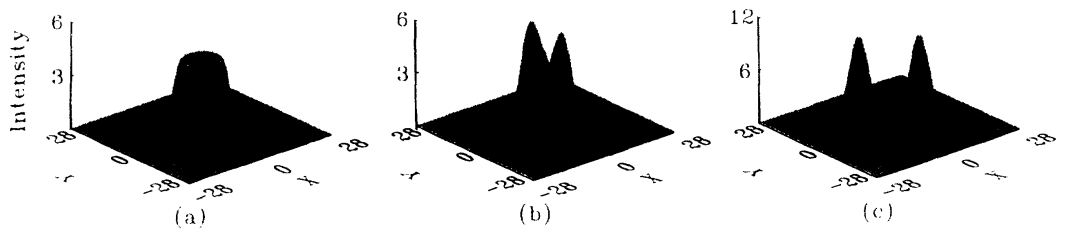

FIG. 2. Dynamical evolution of the fundamental bound state for $\gamma^{2}=0.5$ (a) at $Z=0$, (b) at $Z=40$, and (c) at $Z=50$. itive too [see Fig. 1(a) for $\lambda_{2}=\lambda_{\text {as }}$ vs $\gamma^{2}$ ]. This means for an asymmetric ( $\phi$-dependent) perturbation, the largest quantity $\left\langle u \mid L_{11} u\right\rangle$ of Eq. (5) [or the largest eigenvalue of Eq. (6)] can be greater than zero, and real $\delta$ exists. In other words, the fundamental bound state is unstable against asymmetric perturbations. This prediction agrees with the analysis of direct numerical solution to the linearized equation (4a). Figure 1(b) shows the growth rate of the asymmetric perturbations $u=U(r) \cos m \phi$ and $v=V(r) \cos m \phi$ for the $n=1$ state. Over $0<\gamma^{2}<0.88, m=2$, asymmetric perturbation dominates, whereas $m=1$ ("snake-type" [19]) instability prevails when $\gamma^{2}>0.88$. The dashed curves shown in Fig. 1(b) are the growth rates predicted by the approximate analytical expression

$$
\delta=\delta_{n l m}=\left[\frac{m^{2}+1}{\bar{r}_{n l}^{2}}\left(\frac{2 \bar{\psi}_{n l}^{2}}{\left(1+\bar{\psi}_{n l}^{2}\right)^{2}}-\frac{m^{2}+1}{\bar{r}_{n l}^{2}}\right)\right]^{1 / 2}
$$

where $\bar{Q}_{n l}=\int_{r_{l}}^{r_{l+1}} r Q \psi_{n}^{2} d r / \int_{r_{l}}^{r_{l+1}} r \psi_{n}^{2} d r$ with $Q=\psi$ or $r$, and $r_{j}$ is the $j$ th zero of the $n$th bound state. This approximation, as in the case of the self-trapped beams with the maximum intensity at the center [15], gives a reliable evaluation of the growth rate over a wide range of $\gamma^{2}$ (from $\gamma^{2}=0$ up to $\gamma^{2}=0.6$ for $m=2$ and up to $\gamma^{2}=0.9$ for $m=1$ ).

To verify the prediction of the stability characteristics obtained from the linear stability analysis, we conduct numerical simulations of Eq. (2). Figure 2 illustrates the nonlinear dynamics of the fundamental bound state (at $\gamma^{2}=0.5$ ) evolving from the symmetric ring structure with the zero intensity at the center to two filaments which spiral away from the center of the initial state with the propagation distance, agreeing with the linear stability analysis of Fig. 1(b) that predicts that the $m=2$ growth mode predominates at $\gamma^{2}=0.5$. In the numerical simulations a random noise is implanted in the initial input $\left\{\psi(x, y, 0)=\psi_{n}[1+\Gamma(x, y)]\right.$ with the mean value $\langle\Gamma\rangle=0$ and the variance $\left.\left\langle|\Gamma|^{2}\right\rangle=0.0001\right\}$. Without a
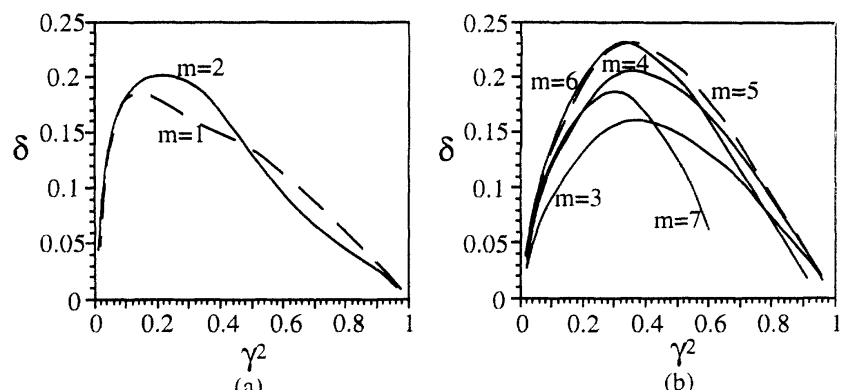

FIG. 3. Dependence of the growth rates $\delta$ of the $n=2$ bound state on $\gamma^{2}$. random noise, the evolution of the beam would follow the same patterns, although at a longer distance.

The analysis for the $n=1$ state above applies to the other higher-order bound states, and shows that all the higher-order states display transverse instabilities as well. The difference is that the nonlinear dynamics of the beam evolution becomes more and more complicated with increasing order $n$. Figure 3 gives the growth rates of the first higher-order $(n=2)$ state vs $\gamma^{2}$. The field profiles of the $m=1$ and 2 growth modes (nearly) coincide with the inner ring field profile of the bound state, and thus they are responsible for the instability development of the inner ring. This is shown in Fig. 4 where two filaments develop from the inner symmetric ring, consistent with the prediction of Fig. 3(a) that the $m=2$ growth mode dominates at $\gamma^{2}=0.2$. On the other hand, the field profiles of $m=3,4,5,6,7$ growth modes locate at the outer ring field profile of the $n=2$ bound state and the growth mode with the largest growth rate among them mainly contributes to the instability development of the outer ring. This is demonstrated in Fig. 3(b) where the $m=6$ growth mode with the largest growth rate (below $\left.\gamma^{2}<0.35\right)$ dominates the instability development, leading to the six filaments (developed from the outer ring) in Fig. 4 at $\gamma^{2}=0.2$. With increasing $n$, more filaments result, and in general for a fixed $n$ and $\gamma$ the number of filaments developed per ring increases with increasing ring radius.

Filaments develop from transverse instabilities. Then what are these filaments? We believe that each of these filaments is a quasi-self-trapped beam pattern with maximum intensity at the center, as discussed in Ref. [9]. In the presence of two or more filaments, the location of filaments will vary with the propagation distance, as a result of the presence of the interaction force among filaments (see Figs. 2 and 4), whereas one filament present alone will stay there without changing its location. The greater the number of filaments, the more complicated the resulting interaction. Take the simplest case of the presence of two filaments in Fig. 2. The interaction force between the filaments pushes the filaments spiralling away from the center (of the bound state), and they distance themselves until they reach the equilibrium state of the individual
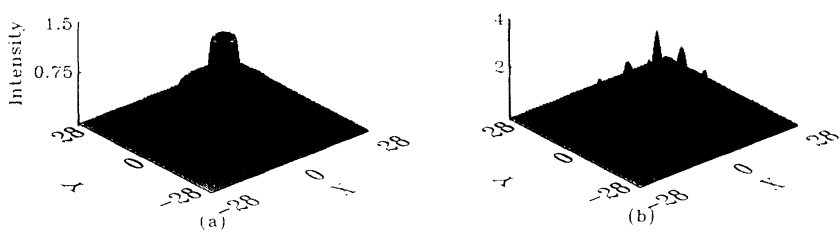

FIG. 4. Dynamical evolution of the first higher-order bound state for $\gamma^{2}=0.2$ (a) at $Z=0$, and (b) at $Z=50$. 
filament (when they are far separated and the interaction between the filaments becomes negligible). A similar development occurs for higher-order states (Fig. 4) where filaments spiral away from the dark spot with increasing distance and finally reach the equilibrium state of the individual filament at a longer distance. The larger the $n$, the longer the distance required for more filaments to be separated.

In conclusion, the stability characteristics of the selftrapped beam with a dark spot surrounded by rings of varying intensity are examined analytically and numerically. We show that the fundamental bound state of the family is stable against symmetric perturbation but un- stable against an asymmetric perturbation. The higherorder states are also found to display transverse (modulation) instabilities. The development of transverse instabilities is shown to lead to the emission of filaments which spiral away from the dark spot and move toward the equilibrium state of the three-dimensional self-trapped beam with maximum intensity at the center.

J.A. acknowledges a grant from the Australian Telecommunications and Electronics Research Board and Y.C. is grateful for financial support from the Australian Research Council. Optical Sciences Centre is a member of Australian Photonics Cooperative Research Centre.
[1] R. Y. Chiao, E. Garmire, and C. H. Townes, Phys. Rev. Lett. 13, 479 (1964).

[2] H. A. Haus, Appl. Phys. Lett. 8, 128 (1966).

[3] P. L. Kelly, Phys. Rev. Lett. 15, 1005 (1965).

[4] E. Garmire, R. Y. Chiao, and C. H. Townes, Phys. Rev. Lett. 16, 347 (1966).

[5] R. Y. Chiao, M. A. Johnson, S. Krinsky, H. A. Smith, C. H. Townes, and E. Garmire, IEEE J. Quantum Electron. QE-2, 467 (1966).

[6] M. M. Denariez, Denariez-Roberge, and J. E. Taran, Appl. Phys. Lett. 14, 205 (1969).

[7] R. R. Alfano and S. L. Shapiro, Phys. Rev. Lett. 24, 592 (1970).

[8] S. A. Akhmanov, R. V. Khokhlov, and A. P. Sukhorukov, in Laser Handbook, edited by F. T. Arecchi and E. O. Schulz-DuBois (North-Holland, Amsterdam, 1972), p. 1151.

[9] N. G. Vakhitiov and A. A. Kolokolov, Izv. Vyssh. Uchebn. Zaved. Radiofiz. 16, 1020 (1973) [Sov. Radio Phys. 16, 783 (1973)].

[10] A. A. Kolokolov and A. I. Sykov, Zh. Prikl. Mekh. Tekh.
Fiz. 4, 56 (1975) [J. Appl. Mech. Tech. Phys. 4, 519 (1975).

[11] Y. R. Shen, Prog. Quantum Electron 4, 1 (1975).

[12] J. H. Marburger, Prog. Quantum Electron 4, 35 (1975).

[13] P. L. Baldeck, F. Raccah, and R. R. Alfano, Opt. Lett. 12, 588 (1987).

[14] H. Zhang, J. Dai, P. Wang, and L. Wu, Opt. Lett. 14, 695 (1989).

[15] J. M. Soto-Crespo, D. R. Heatley, E. M. Wright, and N. N. Akhmediev, Phys. Rev. A 44, 636 (1991); J. M. SotoCrespo, E. M. Wright, and N. N. Akhmediev, ibid. 45, 3168 (1992).

[16] D. I. Abakarov, A. A. Akopyan, and S. I. Pekar, Zh. Eksp. Teor. Fiz. 52, 463 (1967) [Sov. Phys. JETP 25, 303 (1966).

[17] Y. Chen, IEEE J. Lightwave Tech. LT-9, 1208 (1991).

[18] Y. Chen and A. W. Snyder, Electron. Lett. 27, 565 (1991); Y. Chen, IEEE J. Quantum Electron. QE-26, 1236 (1991).

[19] V. E. Zakharov and A. M. Rubenchik, Zh. Eksp. Teor. Fiz. 65, 987 (1973) [Sov. Phys. JETP 38, 494 (1974)]. 


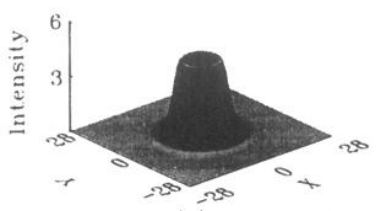

(a)

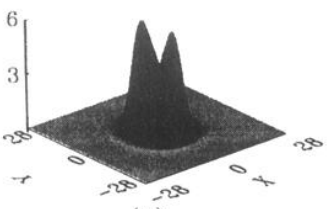

(b)

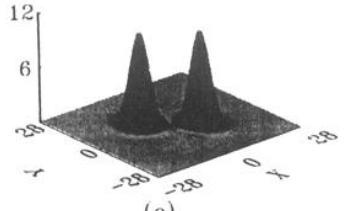

(c)
FIG. 2. Dynamical evolution of the fundamental bound state for $\gamma^{2}=0.5$ (a) at $Z=0$, (b) at $Z=40$, and (c) at $Z=50$. 

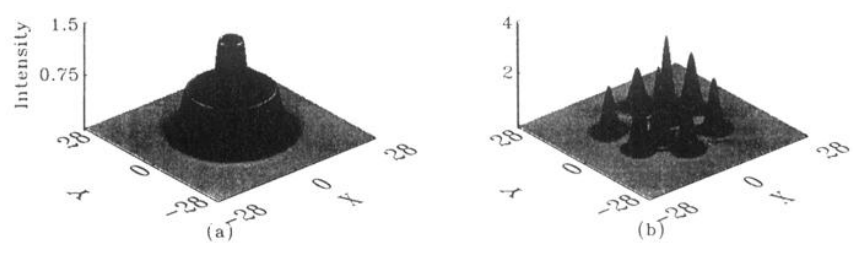

FIG. 4. Dynamical evolution of the first higher-order bound state for $\gamma^{2}=0.2$ (a) at $Z=0$, and (b) at $Z=50$. 